\title{
CHALLENGES TO STATE-OF-THE-ART MOULD LEVEL CONTROL SYSTEMS AND INNOVATIVE SOLUTIONS*
}

\author{
Silvio Cecchini ${ }^{1}$ \\ Pawel Krajewski \\ Sebastian Michelic ${ }^{3}$ \\ Heinz Rumpler ${ }^{4}$ \\ Robert Zirk $1^{5}$ \\ Bernhard Koeller ${ }^{6}$
}

\begin{abstract}
State-of-the Art Mould Level Control Solutions for continuous casting applications face different challenges. On the one hand, technological phenomena such as clogging and unclogging, unsteady bulging or waving can create unpredicted meniscus disturbances by completely changing the casting channel geometry and the flow behaviour of liquid steel to the mould. On the other hand, from the viewpoint of caster operations, long lifetime, quick interchange-ability and integration capability into existing systems must be achieved. In the present contribution, the overall development of INTECO TBR's mould level master - a comprehensive mould level control solution for all continuous casting applications - will be introduced. The basis for the presented system is a development over several years which includes investigations of all critical components such as shroud, stopper, stopper mechanism, servo actuator and mould level control program. These investigations finally resulted in a new-ly developed control algorithm for a highly dynamic servo actuator. The control algorithm with its basic functionality such as the prevention, identification and removal of clogging impacts with the associated automation functions to reduce their influence onto the overall mould level control performance will be presented. The scientific basis, the resulting control functions and their related automation implementation will also be illustrated. Finally, a patented control algorithm and control functionality for unsteady bulging and waving effects with the associated automation functions to reduce their influence onto the overall mould level control performance.
\end{abstract}

Keywords: Continuous casting; Automation; Mould level control; Quality.

Austria.

6 Project Development, INTECO TBR casting technologies GmbH, Leoben, Styria, Austria. 


\section{INTRODUCTION}

Casting of high quality steel grades in the continuous casting process depends from serval metallurgical and process parameters. One of most important of them is a guarantee of stable mould level. Any changes in the mould level may lead to surface and inside defects and in the worst case to breakout. The unstable mould level is a consequence of an agglomeration of deoxidation products, solidified steel in the submerged entry nozzle/ shroud (clogging) or rapid drop oxide from the nozzle (unclogging). The further problems with mould level stability are caused by the molten steel discharge through the submerge entry shroud (SES) into the mould (waving), changes in the strand temperature and lower shell stiffness (unstable bulging) [1-3]. In some cases, waving can be also linked to the mould level reading noise, which moves the stopper without having a real meniscus variation [1]. Changes of the nozzle/shroud and stopper geometry due to the wearing affect also the flow and the mould level. According to mathematical models calculations for conical stoppers a changes of stopper/nozzle geometry, steel level in the tundish or steel density of only $1 \%$ cause the change of the flow of up to $34 \mathrm{l} / \mathrm{min}$. Because of this instability, the guarantee of a stable mould level is a difficult task. A stable mould level is especially a challenge in the case of peritectic and high aluminium steel grades. The guarantee of stable casting conditions can be only possible by using of very precise system to control the mould level fluctuations. The requirement for such as system is very fast reaction (counter reaction) on any changes in the mould level. This requirement can only be met by using a very precise control mechanism. The software algorithm must detect and identify the type of any periodic and non-periodic variation in the mould level fluctuation and reacts as quickly as possible. The algorithm has to detect the mould level fluctuation and counter reaction of the stopper to improve the stability of mould level in the further CC process.

INTECO TBR Casting Technologies developed a high tech system to control mould level fluctuations. This system includes the precise stopper rod mechanism with servo actuator and software control program: Mould Level Master (MLM). The following paper describes the whole package and gives practical examples of functionality the new system.

\section{STOPPER ROD MECHANISM AND SERVO ACTUATOR}

The stopper rod mechanism and servo actuator regulate directly the steel flow from the tundish to the mould. The stopper rod mechanism geometry depends on the cast format, respectively the length of the gooseneck. The stopper rod mechanism of INTECO TBR is based on long time experience of stopper mechanism design which is shown by the number of world-wide installations. The whole equipment is presented in Figure 1. The most important points in this mechanism is a precise and adjustable guiding. By special design of the gooseneck controlled bending and deformation during the casting operation can be avoided. 


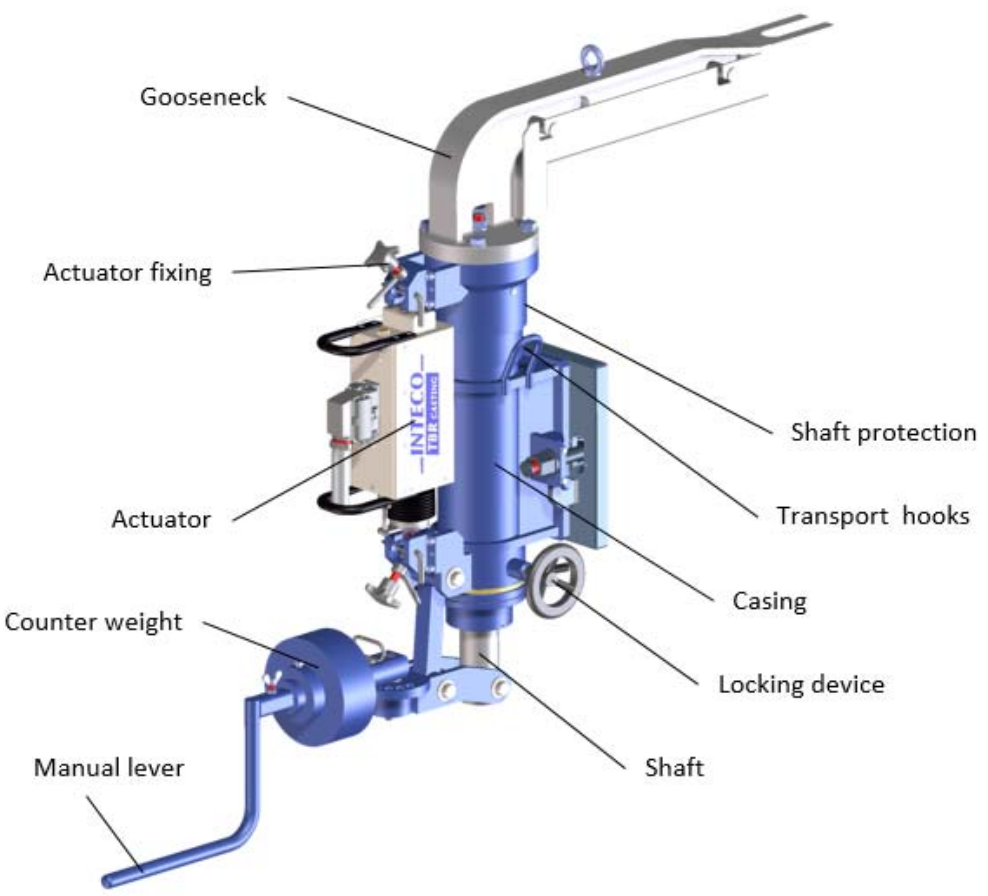

Figure 1. Stopper rod mechanism with servo actuator

The servo actuator (Figure 1) is an electromechanical motor which is mounted on the stopper rod mechanism. It is controlled by the Mould Level Master and performs the movements with highest accuracy. The servo actuator is characterized by fast acting and precise control. One further advantage is small size and low weight $(19 \mathrm{~kg})$.

\section{MOULD LEVEL MASTER}

The Mould Level Master MLM is the software package to control the steel flow to the mould. The principle of the MLM is presented in the Figure 2. The steel level in the mould is measured by the level sensor mounted in the mould. The processed signal is sent to the MLM. The software checks the set mould position with actual position of the mould level. In the case of any differences, the MLM send the signal to the servo actuator to the change of the stopper position. Each change of casting conditions is immediately recognized by the MLM and counter reaction is initiated. The information like: set-point, actual mould level, stopper position and casting speed are visualized at the monitor in the control room. Even though the casting speed is no input parameter into the MLM, any changes of casting speed by the process or operator will be immediately detected and the servo actuator will react to stabilize the mould level under new conditions. The major highlights of the MLM are the limited level fluctuations with $<+/-2 \mathrm{~mm}$ and its short lag time of app. $15 \mathrm{~ms}$. 


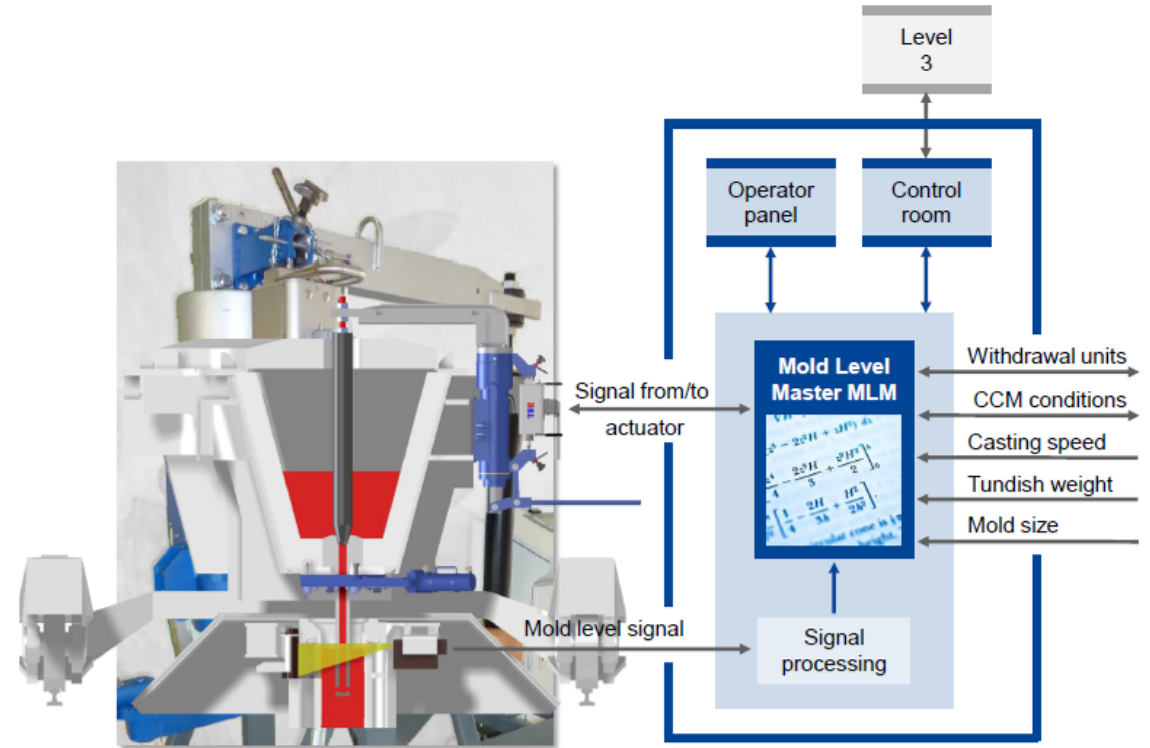

Figure 2. The principle of Mould Level Master

The MLM has the following technological functionalities to stabilize the mold level:

\subsection{Bulging and Waving Detection and Counter - Reaction}

These features can be only competed due to high precision algorithm, which detects any variation in the mould level fluctuation and reacts of them. The algorithm detected two typical types of variations: non-periodic variations in the case of mould level with small fluctuation due to e.g. clogging/unclogging and long periodic variation in the case of waving and unsteady bulging. The differences in variations are clearly seen in the Figure 3. Waving characterizes by the higher frequency of mould level fluctuations in compared to unsteady bulging.

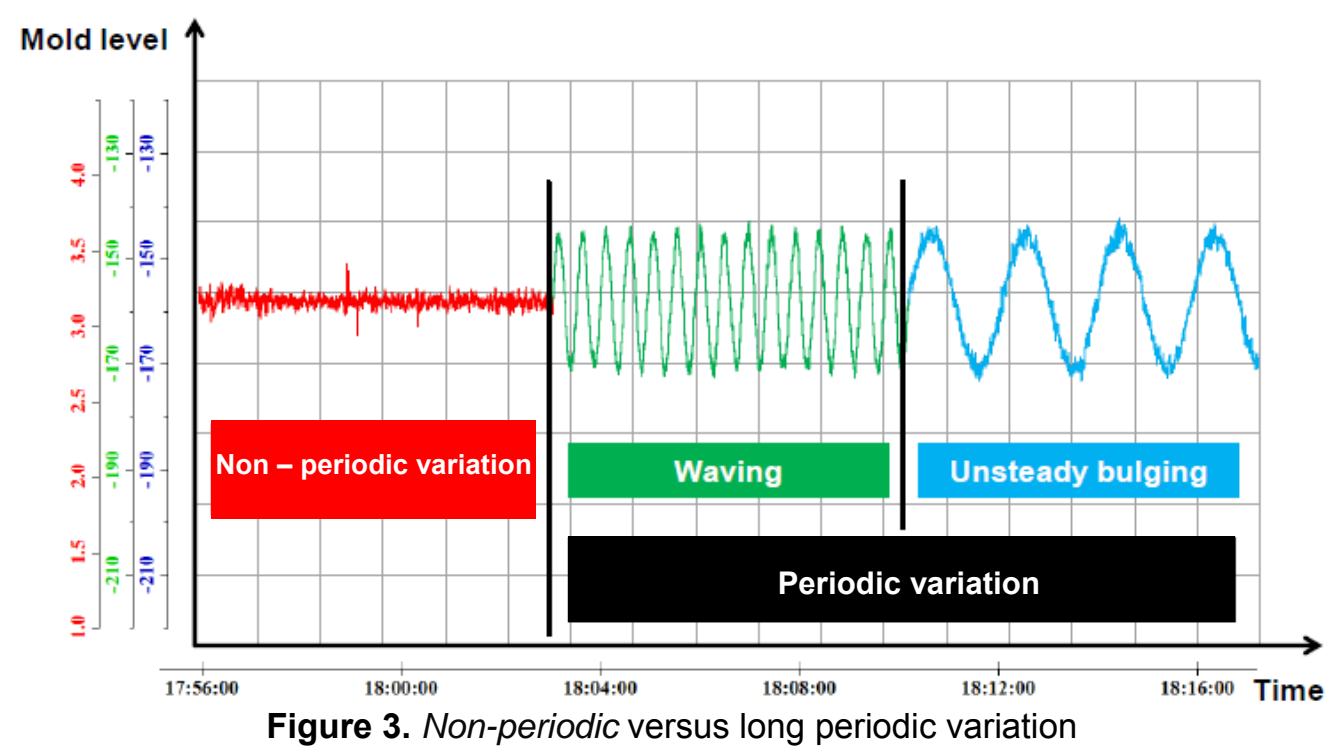

Two detection logics are implemented in the algorithm - one for waving, one for bulging - both of them running continuously and analyzing the mould level. The implemented algorithm finds the right settings to minimize the deviation of mould level whatever the type of variation. The period of disturbances, generated by casting conditions, is 
shorter than the one of the unsteady bulging or waving, which means that a precise discrimination, between the different disturbances, can be carried out [1]. The algorithm detecting periodic distributions is presented in Figure 4.

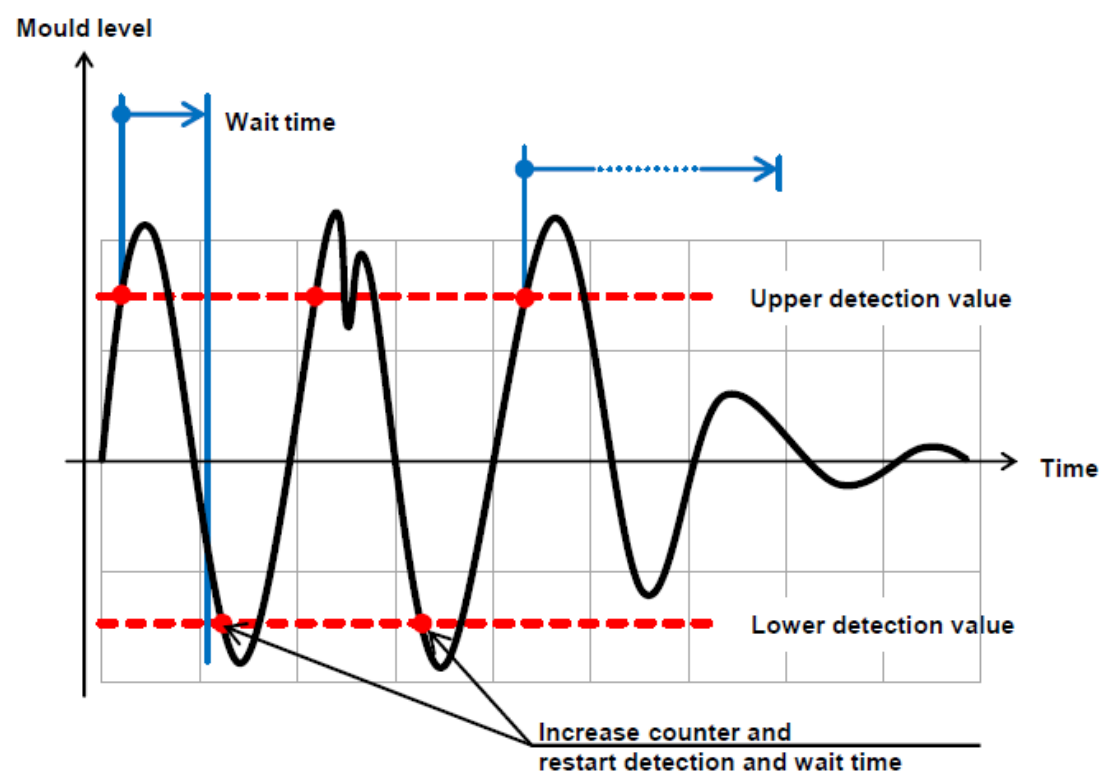

Figure 4. Counting method for bulging and waving

\subsection{Clogging Detection and Counter Reaction}

Clogging decreases the steel flow to the mould. The reaction of MLM in the case of clogging is moving up the stopper to increase the steel flow. However if the counter reaction is not sufficient the MLM has two options in the case of clogging detection. It will be either anti-clogging or flushing. In the case of the first function, the stopper vibrates to reduce the clogging behavior. Due to the high vibration frequency, the steel flow variation average is zero and therefore does not affect the mould level stability. If the flushing function is initiated, stopper position is memorized, than the stopper closes fully, in order to mechanically remove the clogging build-up by mechanical impact.

In the case of bulging, waving or clogging detection, the counter reaction can be manually, semi-manually or full automatic. Each of the counter reaction depending from the detected problem has own procedure implemented in MLM.

\subsection{Reduced Refractory Wear}

To increase the life-time of the submerged entry shroud the mould level set-point moves around its set value in order to distribute the SES wear. This functionality does not interfere with the stability of the level.

\subsection{Protection in the Case of Too High/Low Mould Level}

The MLM is additionally equipped with an automatic protection in the case of too high/low steel level in mould. If the level exceeds a predefined high level limit for a defined time or the mould level drastically drops below a certain set point, an automatic protection is immediately initiated. This option avoids the steel overflow in the mould and breakout. 


\section{PRACTICAL EXAMPLE}

A practical example was performed at the two-strand slab continuous casting machine in Germany. The aim of the investigation was to demonstrate the functionality of INTECO TBR mould control system and compare it with the standard system used in the company. While strand \#1 was equipped with the standard mould control system and hydraulic mechanism used normally by the company, strand \#2 was connected to the INTECO TBR test equipment including MLM and servo actuator.

The test equipment with control panel can be quickly installed at each continuous casting machine. The test equipment is presented in the Figure $5 \mathrm{a}$. The servo actuator was installed on the existing stopper rod mechanism (Figure 5b).
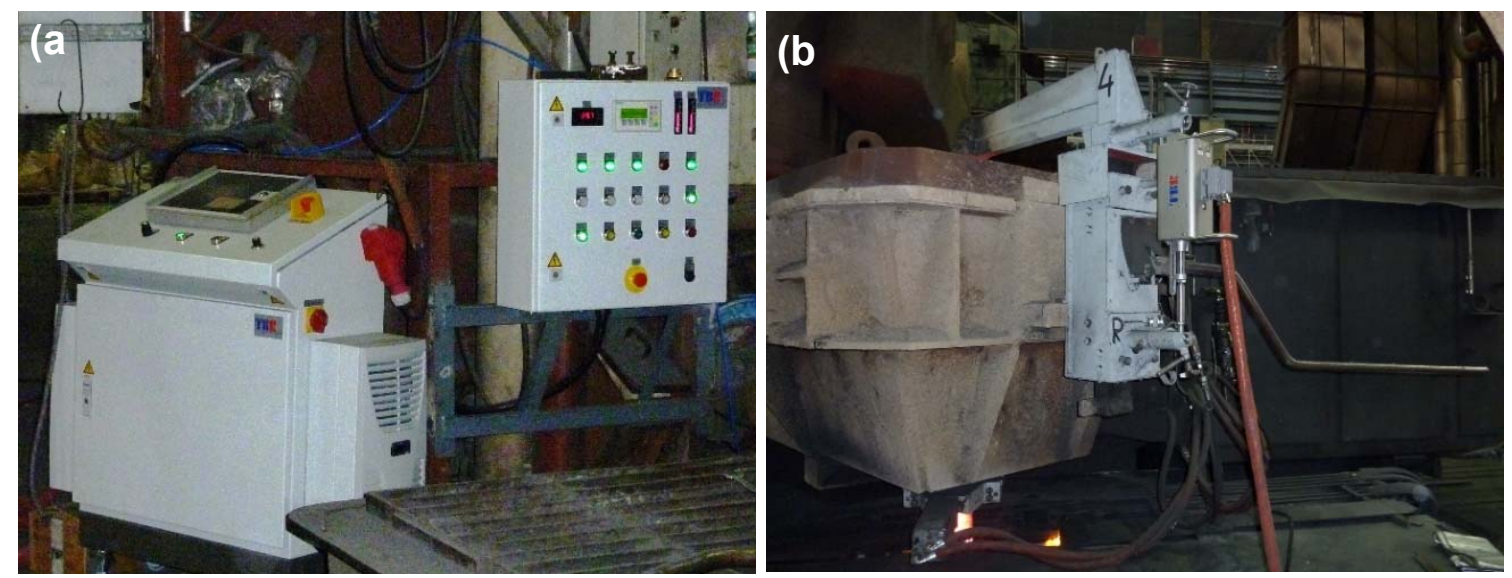

Figure 5. (a) test equipment with control panel, (b) servo actuator installed on the existing stopper rod mechanism

The mould level at the both strand were measured with $\mathrm{VUHZ}$ sensor. The experiment were performed in one week by casting of 43 ladles.

Figure 6 presents the results of the mould level fluctuation on both strands in the period of 20 minutes. The upper picture shows the results for the standard system and lower for MLM. Results show that the mould level fluctuations on the first strand are very high even with a constant casting speed. The stopper react very slowly and cannot stop the fluctuations. The Inteco TBR mould level control is much more stable. Fluctuations occurs only $+/-1.5 \mathrm{~mm}$. Even small changes in the casting speed does not affect the level stability. The stopper is very active and reacts very quickly of any fluctuation.

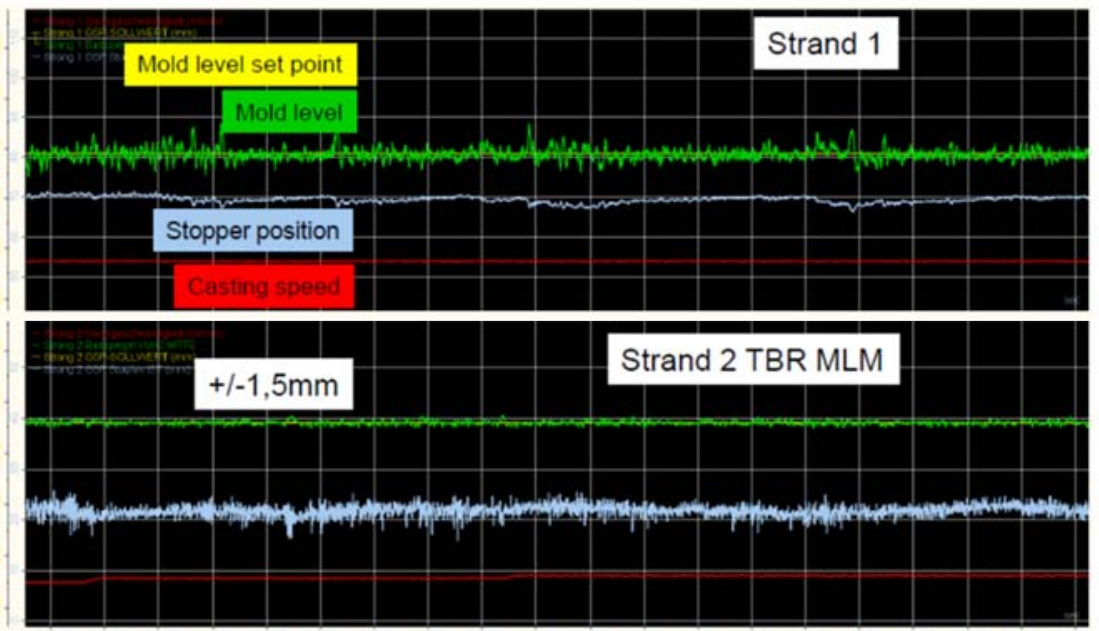

Figure 6. Comparison of the mould level control behavior for Strand $1 \& 2$. 
One more weakness of the standard mould level control system can be seen in the Figure 7 . Both strand were affected by clogging at the same time. The mould level was far away from the set point in the standard system. The control program detected that the flow in the submerged entry nozzle decreases and moved up the stopper. The reaction was too slow and not effective. The calculated necessary stopper position was too high. The standard system loosed completely control at the mould level. The operator had to stop the strand withdrawal and decreased the casting speed to zero to stabilize the mould level. The system did not switch on either anti-clogging or flushing option to counteract the clogging. The system developed by INTECO TBR showed a more stable behaviour. The MLM recognizes very quickly clogging and move up the stopper. The stopper opening is not so rapid according to the necessary counter reaction calculated by MLM.

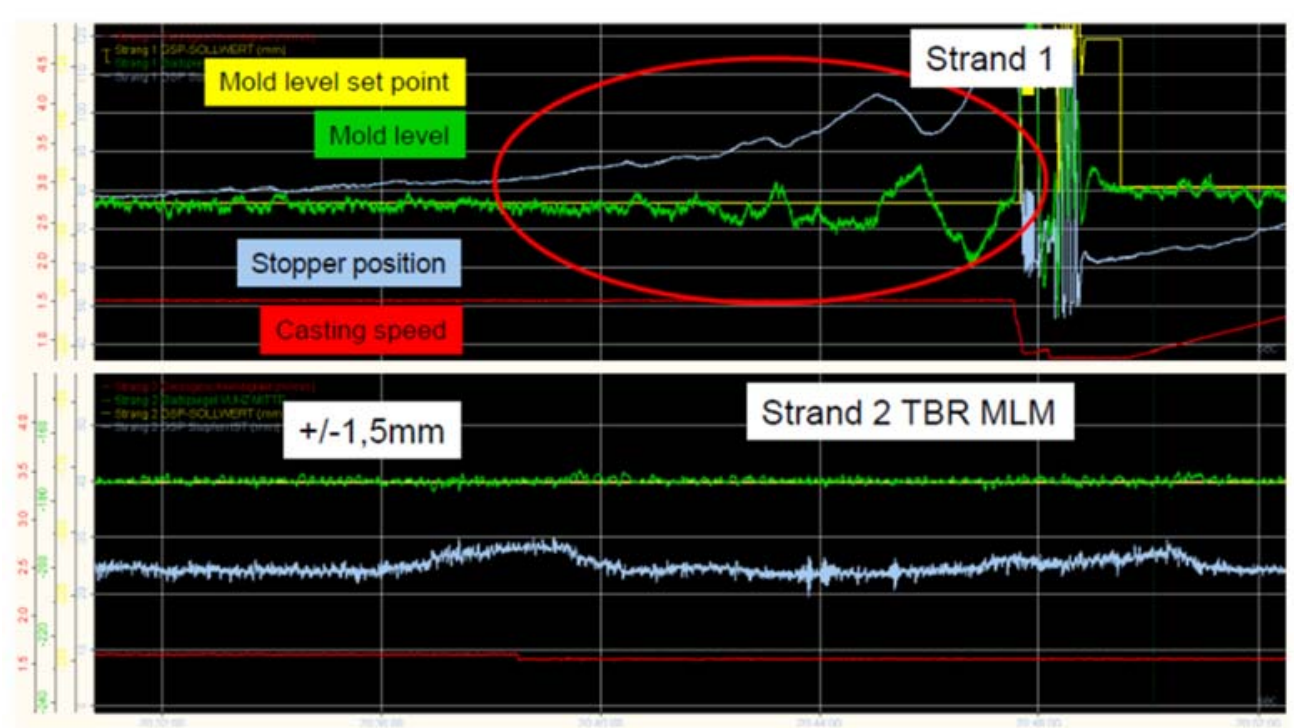

Figure 7. Example of clogging and reaction of stopper and effect on mould level

The INTECO TBR servo actuator used was only slightly loaded during the entire experimental period and showed no signs of wear or other abnormalities. The temperature of servo actuator increased only of $5-8^{\circ} \mathrm{C}$ above the surrounding temperature $\left(45^{\circ} \mathrm{C}\right)$ in the time of $6 \mathrm{~h}$ without any additional cooling.

\section{SUMMARY}

The new INTECO TBR mould level control is high precise system, which guarantees a very stable mould level of $+/-2 \mathrm{~mm}$. The highlight of this control system is very active stopper, which moves quickly up and down depending from the necessary reaction (counter reaction). The Mould Level Master determines the type of counter reaction and the stopper position, depending from type of variation.

The electromechanic servo-actuator designed by INTECO TBR allows a precise movement and quick response time. The possibility of installed the servo actuator on existing stopper rod mechanism, small size and weight are additional advantages of the system. 


\section{REFERENCES}

1 Cechini S., R. Zirkl and M. Pillwax: Bulging and Waving - Difficult for Automatic Mold Level Control, Conference Paper, AISTech 2013.

2 Bernhard C. und A. Karasangabo: Einschlussmodifikation - Beispiele für die qualitätsbestimmende Bedeutung von NME, Vorlesung, Montanuniversität Leoben, 2012.

3 Lee J. D and C. H. Yim: The mechanism of unsteady bulging and its analysis with the finite element method for continuously cast steel, ISIJ International, 40 (2000) 8, 765770 . 